\title{
Birth defects after use of antithyroid drugs in early pregnancy: a Swedish nationwide study
}

\author{
Stine Linding Andersen 1,2, Stefan Lönn ${ }^{3}$, Peter Vestergaard ${ }^{1,4}$ and Ove Törring ${ }^{5,6}$ \\ ${ }^{1}$ Departments of Endocrinology and ${ }^{2}$ Clinical Biochemistry, Aalborg University Hospital, Aalborg, Denmark, \\ ${ }^{3}$ Department of Research and Development, Region Halland, Halmstad, Sweden, ${ }^{4}$ Department of Clinical Medicine, \\ Aalborg University, Aalborg, Denmark, ${ }^{5}$ Department of Clinical Research and Education, Södersjukhuset, Karolinska \\ Institutet, Stockholm, Sweden, and 'Division of Endocrinology, Department of Internal Medicine, Södersjukhuset, \\ Stockholm, Sweden
}

Correspondence should be addressed to S L Andersen Email

stine.a@rn.dk

\begin{abstract}
Objective: Antithyroid drugs (ATDs) may have teratogenic effects, but more evidence is needed on the risk and types of birth defects after the use of methimazole (MMI) and propylthiouracil (PTU). This study aimed to evaluate the association between the use of ATDs in early pregnancy and birth defects.

Design: Swedish nationwide register-based cohort study.

Methods: The study included 684340 children live-born in Sweden from 2006 to 2012. Exposure groups defined by maternal ATD use in early pregnancy were MMI $(n=162)$; PTU $(n=218)$; MMI and PTU $(n=66) ;$ ATD before or after, but not in pregnancy $(n=1551)$ and non-exposed (never ATD $(n=682343)$ ). Outcome was cumulative incidence of birth defects diagnosed before two years of age.

Results: The cumulative incidence of birth defects was not significantly different in children exposed to MMI $(6.8 \%, P=0.6)$ or PTU $(6.4 \%, P=0.4)$ vs non-exposed $(8.0 \%)$. For subtypes of birth defects, MMI was associated with an increased incidence of septal heart defects $(P=0.02)$. PTU was associated with ear $(P=0.005)$ and obstructive urinary system malformations $(P=0.006)$. A case of choanal atresia was observed after exposure to both MMI and PTU. The incidence of birth defects in children born to mothers who received ATD before or after, but not in pregnancy, was $8.8 \%$ and not significantly different from non-exposed $(P=0.3)$, MMI exposed $(P=0.4)$ or PTU exposed $(P=0.2)$. Conclusions: MMI and PTU were associated with subtypes of birth defects previously reported, but the frequency of ATD exposure in early pregnancy was low and severe malformations described in the MMI embryopathy were rarely observed.
\end{abstract}

\section{Introduction}

Hyperthyroidism in pregnant women should be adequately treated to prevent maternal and fetal complications $(1,2,3)$. The treatment of choice in pregnancy is antithyroid drugs (ATDs) $(4,5)$. A main concern about the use of ATDs in general is the risk of severe side effects such as agranulocytosis and liver failure (6). However, for the use of ATDs in pregnant women, a potential risk of birth defects is added to this concern (6).

From the 1970s, an increasing number of case reports described similar types of malformations in children
() 2017 European Society of Endocrinology Printed in Great Britain born to mothers who had been treated with methimazole (MMI) or the prodrug carbimazole (CMZ) in the early pregnancy (7). In more recent years, a number of cohort studies have been published $(8,9,10,11,12,13)$. The majority of studies found no association between birth defects and the use of MMI $(8,10,12,13)$, whereas two studies from Japan (9) and Denmark (11) found that the use of MMI in early pregnancy was associated with 2-4\% additional cases of birth defects compared with non-exposed. Furthermore, the types of birth defects

Published by Bioscientifica Ltd. 
were in line with the previous observations from case reports.

The majority of studies $(8,9,10,12,13)$ did not observe an increased prevalence of birth defects in children born to mothers treated with PTU in the early pregnancy. However, the use of PTU was associated with a higher prevalence of face and neck and urinary system malformations in a Danish nationwide study (11). Such malformations were not observed after MMI, and although the defects appeared less severe, the majority of children underwent surgery (14).

Recently revised clinical practice guidelines from the American Thyroid Association (ATA) recommend the use of PTU in early pregnancy, but also address the possibility of ATD withdrawal in appropriately selected patients $(4,5)$. Many clinical aspects are still uncertain and more studies are needed on the association between the use of ATDs in early pregnancy and birth defects.

A unique feature in the Nordic European countries is the collection of health data in nationwide registers. We used data from Swedish national registers to study the association between the use of ATDs in early pregnancy and birth defects. More specifically, the study aimed to evaluate the previous findings from Denmark in another cohort using the same type of data and a similar design.

\section{Subjects and methods}

\section{Study population}

Swedish health care data are collected in nationwide registers and a unique personal identification number enables linkage between the different registers in encrypted form. In the Swedish Medical Birth Registry (MBR) (15), we identified all live-born children in Sweden between January 1, 2006 and December 31, $2012(n=738$ 502 ) and their mothers as well as information on maternal characteristics (age, parity, type of pregnancy (singleton/ multiple), origin, smoking, height and weight) and child characteristics (gender, gestational age at birth, birth weight, mode of delivery) registered at the time of the woman's midwife consultation in early or late pregnancy or at the time of birth of the child.

\section{Exposure}

Information on maternal use of ATDs was obtained from the Swedish Prescribed Drug Register (SPDR) (16), which holds data on all prescriptions drugs in Swedish since 2005 including the type of drug according to the
Anatomical Therapeutic Chemical classification system (ATC) and the date of sale. We identified all prescriptions of ATDs (ATC H03B) and thyroid hormones (ATC H03A) dispensed between July 1, 2005 and December 31, 2014. ATDs included MMI (H03BB02) and PTU (H03BA02), whereas no prescriptions of carbimazole (H03BB01) were identified.

Pregnancy period (first day of last menstrual period to the birth of the child) was estimated by subtracting gestational age at birth from the date the child was born. We defined the child as exposed to maternal ATD in early pregnancy if the mother had at least one redeemed prescription of ATD less than six months before the estimated pregnancy start and before the 11th gestational week $(n=454)$. In six pregnancies, the mother redeemed prescriptions of ATD in the six-month period before pregnancy, but underwent thyroid surgery before conception. These women redeemed prescriptions of thyroid hormones after the date of thyroid surgery and were not included. Similarly, two women were excluded because they redeemed prescriptions of both MMI and PTU in the six-month period before pregnancy, but initiated continuous treatment with thyroid hormone before pregnancy. Thus, 446 children were classified as ATD exposed and grouped according to the type of maternal ATD treatment: 'PTU exposure' (PTU prescriptions only $(n=198)$ or MMI in the beginning of the period changing to PTU before the estimated pregnancy start $(n=20))$, 'MMI exposure' (MMI prescriptions only $(n=162)$ ), and 'MMI and PTU exposure' (both MMI and PTU treatment after the estimated pregnancy start; MMI followed by PTU $(n=64)$, PTU followed by MMI $(n=2)$ ).

Of the children who were not exposed to ATD, we predefined a group of children $(n=1551)$ born to mothers who were treated with ATD more than one year before or more than one year after pregnancy and received no treatment with thyroid hormone in pregnancy. Finally, children born to mothers with no redeemed prescriptions of ATD or thyroid hormones from 2005 to 2014 and no diagnosis of hyperthyroidism registered from 2005 to 2014 in the Swedish National Patient Register (SNPR) (17) were categorized as 'non exposed' ( $n=682343)$. Children who did not fulfill the criteria for any of the exposure groups were not included (7.3\%), and the majority were excluded from the non-exposed group, because the mother had redeemed prescriptions of thyroid hormones.

To validate the frequency of ATD exposure in early pregnancy comparison was made to the frequency of ATD exposure in a Danish nationwide study population (11). For comparison, we obtained nationwide information on 
redeemed prescriptions of ATDs in women of childbearing age (20-39 years) in Sweden (retrieved March 3, 2017 from www.socialstyrelsen.se/statistik/statistikdatabas/ lakemedel) and in Denmark (retrieved March 3, 2017 from www.medstat.dk).

\section{Outcome}

Diagnoses of birth defects in the children were obtained from the SNPR (17), which holds nationwide data on both inpatient and outpatient visits to any Swedish hospital and specialist clinics since 2001 coded according to the 10th International Classification of Disease (ICD-10). We included all inpatient and outpatient visits with a main or additional diagnosis of birth defects (ICD-10: Q00-Q99) registered before the child was aged two years. The SNPR did not include diagnoses from general practice.

\section{Statistical analysis}

The primary outcome was predefined as a diagnosis of one or more birth defects (all types combined) before the child was aged two years. The secondary outcome was 13 predefined subgroups of malformations according to ICD-10 classification, as previously described (11). Based on previous findings (18), we also selected the specific diagnoses of heart septal defects as outcome (ICD-10: Q21). Chi-square test was used to compare the cumulative incidence (referred to as incidence) of birth defects by exposure groups, and the Mann-Whitney $U$ test was used for comparison of the time interval from pregnancy start to the shift in therapy from MMI to PTU in early pregnancy.

To evaluate the role of potential confounding, we used a logistic regression model to estimate crude and adjusted odds ratio with 95\% confidence interval for the overall incidence of birth defects. The adjusted model included birth year of the child, maternal age, parity, type of pregnancy, body mass index, smoking and origin as categorical variables. Additionally, information was obtained on maternal comorbidities (registered before, during or up to two years after pregnancy) including diabetes (hospital diagnosis in SNPR (ICD-10: E10-E14) or redeemed prescriptions of antidiabetic drugs in SPDR (ATC: A10)) and use of antiepileptic drugs (ATC: N03).

The study was approved by the Regional Ethical Review Board in Stockholm, Sweden (April 5, 2015; Dnr 2015/3:8) and the National Board of Health and Welfare in
Sweden (August 19, 2015; Dnr 24152/2015). All data were made available in encrypted form so that no individuals could be identified.

Statistical analyses were performed using STATA, version 14 (Stata Corp). A 5\% level of significance was chosen.

\section{Results}

Altogether 684340 children were included and 446 children $(0.065 \%)$ were exposed to maternal use of ATD in early pregnancy (PTU 0.03\%, MMI 0.02\%, MMI and PTU $0.01 \%)$. PTU treatment was more common than MMI, and ATD treatment in early pregnancy was less frequent in the Swedish population than previously observed in the Danish population. Comparison of the overall use of ATD in women age 20-39 years in the two Nordic countries corroborated this disparity (Fig. 1).

As expected, women treated with ATD in pregnancy were older and more frequently smoking compared with the large group of non-exposed children (Table 1). In addition, they were more often of non-Swedish origin (Table 1). The study also included 1551 children born to mothers treated with ATD before or after, but not in pregnancy (Table 1 ) and the majority of these women $(n=1408)$ were treated with ATD in the years after pregnancy.

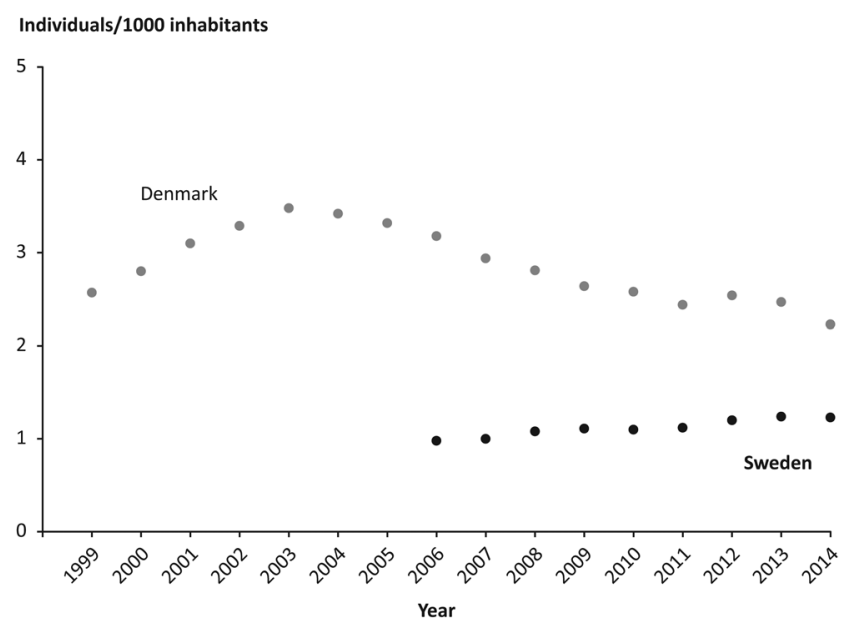

Figure 1

Use of antithyroid drugs as evaluated from redeemed prescriptions of drugs in women aged 20-39 years in Sweden and Denmark. Data were only available in Sweden from the year 2006 and onward. 
Table 1 Maternal and child characteristics in the study population.

\begin{tabular}{|c|c|c|c|c|c|c|}
\hline & \multicolumn{2}{|c|}{ ATD in early pregnancy } & \multicolumn{2}{|c|}{ ATD before or after pregnancy } & \multicolumn{2}{|c|}{ No ATD from 2005 to 2014} \\
\hline & $n$ & $\%$ & $n$ & $\%$ & $n$ & $\%$ \\
\hline Children & 446 & & 1551 & & 682343 & \\
\hline \multicolumn{7}{|l|}{ Maternal characteristics } \\
\hline \multicolumn{7}{|l|}{ Age (years) } \\
\hline$<25$ & 42 & 9.4 & 203 & 13.1 & 88459 & 13.0 \\
\hline $25-29$ & 114 & 25.6 & 440 & 28.4 & 187309 & 27.4 \\
\hline $30-34$ & 148 & 33.2 & 536 & 34.5 & 240129 & 35.2 \\
\hline $35-39$ & 112 & 25.1 & 298 & 19.2 & 134588 & 19.7 \\
\hline$\geq 40$ & 30 & 6.7 & 74 & 4.8 & 31858 & 4.7 \\
\hline \multicolumn{7}{|l|}{ Parity } \\
\hline Nulliparous & 159 & 35.7 & 692 & 44.6 & 300149 & 44.0 \\
\hline Multiparous & 287 & 64.3 & 859 & 55.4 & 381.194 & 56.0 \\
\hline \multicolumn{7}{|l|}{ Type of pregnancy } \\
\hline Singleton & 434 & 97.3 & 1509 & 97.3 & 663680 & 97.3 \\
\hline Multiple & 12 & 2.7 & 42 & 2.7 & 18663 & 2.7 \\
\hline \multicolumn{7}{|l|}{ Body mass index $\left(\mathrm{kg} / \mathrm{m}^{2}\right)^{\mathrm{a}, \mathrm{c}}$} \\
\hline$<18.5$ & 15 & 3.6 & 42 & 2.9 & 15331 & 2.4 \\
\hline $18.5-24.9$ & 236 & 57.6 & 833 & 58.6 & 382087 & 60.9 \\
\hline$\geq 25$ & 159 & 38.8 & 547 & 38.5 & 230380 & 36.7 \\
\hline \multicolumn{7}{|l|}{ Smoking ${ }^{b, c}$} \\
\hline Yes & 91 & 20.9 & 331 & 22.3 & 108001 & 16.5 \\
\hline No & 344 & 79.1 & 1151 & 77.7 & 545251 & 83.5 \\
\hline \multicolumn{7}{|l|}{ Originc } \\
\hline Sweden & 368 & 82.7 & 1290 & 83.7 & 593337 & 87.4 \\
\hline Other Nordic countries & 14 & 3.1 & 26 & 1.7 & 12142 & 1.8 \\
\hline Outside Nordic countries & 63 & 14.2 & 225 & 14.6 & 73326 & 10.8 \\
\hline \multicolumn{7}{|l|}{ Child characteristics } \\
\hline \multicolumn{7}{|l|}{ Gender } \\
\hline Boy & 234 & 52.5 & 778 & 50.2 & 350598 & 51.4 \\
\hline Girl & 212 & 47.5 & 773 & 49.8 & 331744 & 48.6 \\
\hline \multicolumn{7}{|l|}{ Mode of delivery } \\
\hline Cesarean section & 86 & 19.3 & 282 & 18.2 & 118741 & 17.4 \\
\hline Vaginal delivery & 360 & 80.7 & 1269 & 81.8 & 563602 & 82.6 \\
\hline \multicolumn{7}{|c|}{ Gestational age (weeks + days) } \\
\hline$<37+0$ & 20 & 4.5 & 91 & 5.9 & 39326 & 5.8 \\
\hline $37+0$ to $41+6$ & 392 & 87.9 & 1374 & 88.6 & 598533 & 87.7 \\
\hline$\geq 42+0$ & 34 & 7.6 & 86 & 5.5 & 44484 & 6.5 \\
\hline \multicolumn{7}{|l|}{ Birth weight (grams) ${ }^{c}$} \\
\hline$<2500$ & 15 & 3.4 & 70 & 4.5 & 27916 & 4.1 \\
\hline$\geq 2500$ & 430 & 96.6 & 1480 & 95.5 & 653558 & 95.9 \\
\hline
\end{tabular}

ATD, antithyroid drug.

${ }^{a}$ Calculated from maternal weight in early pregnancy at the first pregnancy midwife consultation.

bSelf-reported information on smoking three months before pregnancy, in early and/or in late pregnancy.

'Children with missing value on maternal body mass index $(n=54710)$, smoking $(n=29171)$, origin $(n=3549)$ or birth weight $(n=871)$ were not included.

\section{Cumulative incidence of birth defects}

The primary outcome under study was the cumulative incidence of birth defects in the child diagnosed before the age of two years. The overall incidence was $8.0 \%$ in the large group of non-exposed children (Table 2). The incidence was not significantly different from non-exposed in children exposed to MMI $(P=0.6)$ or PTU $(P=0.4)$ in the early pregnancy, and the incidence of birth defects after MMI (6.8\%) and PTU (6.4\%) exposure were similar $(P=0.9)$. The overall incidence of birth defects in children born to mothers treated with ATD before or after, but not in pregnancy, was $8.8 \%$ and not significantly increased compared with non-exposed children $(P=0.3)$. The results of the crude and adjusted logistic regression analyses were not considerably different for the overall outcome of birth defects (Table 3). Additional adjustment for maternal comorbidities as well as deviations in birth weight, gestational age at birth and mode of delivery did not change these figures.

The secondary outcome was the subtypes of birth defects according to 13 predefined groups (Table 2). 
Table 2 Cumulative incidence of birth defects diagnosed before the age of two years in children born to mothers treated with methimazole (MMI) or propylthiouracil (PTU) in early pregnancy, in children born to mothers treated with antithyroid drugs (ATD) before or after, but not in pregnancy, and in children born to mothers with no use of ATD in the study period.

\begin{tabular}{l}
\hline \\
Children ( $n$ ) \\
Children with birth defect(s) (Q00-Q99) \\
according to ICD-10, $n$ (\%) \\
Children diagnosed with birth defect(s) \\
according to ICD-10 subgroups, $n$ (\%) \\
Nervous system (Q00-07) \\
Eye (Q10-15) \\
Ear (Q16-17) \\
Other malformations of face and neck (Q18) \\
Circulatory system (Q20-28) \\
Respiratory system (Q30-38) \\
Digestive system (Q39-45) \\
Genital organs (Q50-56) \\
Urinary system (Q60-Q64) \\
Musculoskeletal system (Q65-78) \\
Other malformations of musculoskeletal \\
system (Q79) \\
Integumentary system including breast \\
malformations (Q80-84) \\
Others (Q85-99)
\end{tabular}

\begin{tabular}{c}
\hline $\begin{array}{c}\text { MMI in early } \\
\text { pregnancy }\end{array}$ \\
\hline 162 \\
$11(6.79)$
\end{tabular}

\begin{tabular}{c}
$\begin{array}{c}\text { PTU in early } \\
\text { pregnancy }\end{array}$ \\
\hline 218 \\
$14(6.42)$
\end{tabular}

\begin{tabular}{c}
$\begin{array}{c}\text { ATD before or } \\
\text { after pregnancy }\end{array}$ \\
\hline $\begin{array}{c}1551 \\
136(8.77)\end{array}$
\end{tabular}

\begin{tabular}{ccc}
\hline $\begin{array}{c}\text { No ATD from } \\
2005 \text { to } 2014\end{array}$ & & $P^{a, b}$ \\
\hline $\begin{array}{c}682343 \\
n\end{array}$ & & \\
$54827(8.04)$ & & 0.525
\end{tabular}$$
\begin{gathered}
0 \\
<3(1.23)
\end{gathered}
$$$$
0
$$$$
0
$$

$<3(0.62)$

0

$<3(0.62)$

$<3(1.23)$

0

$$
\begin{gathered}
<3(0.46) \\
0 \\
<3(0.92) \\
0 \\
3(1.38)
\end{gathered}
$$

0

$<3(0.92)$

$<3(0.92)$

3 (1.38)

4 (1.83)

0

$$
\begin{gathered}
<3(0.13) \\
4(0.26) \\
0 \\
5(0.32) \\
38(2.45) \\
18(1.16) \\
4(0.26) \\
25(1.61) \\
7(0.45) \\
32(2.06) \\
<3(0.06) \\
9(0.58)
\end{gathered}
$$

0

$<3(0.62)$

0

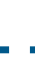

$11(0.71)$

\section{$1018(0.15) \quad 0.640$}

$3134(0.46) \quad 0.211$

$1166(0.17) \quad 0.018$

$1593(0.23) \quad 0.702$

$12981(1.90) \quad 0.132$

$7927(1.16) \quad 0.395$

$1765(0.26) \quad 0.253$

$9415(1.38) \quad 0.220$

$2846(0.42) \quad 0.170$

$14310(2.10) \quad 0.881$

$535(0.08) \quad 0.953$

$2826(0.41) \quad 0.455$

$2443(0.36)$

0.093

ICD-10: 10th International Classification of Disease.

'Result of chi-square test: 'MMI in early pregnancy' vs 'PTU in early pregnancy' vs 'ATD before or after pregnancy' vs 'No ATD from 2005 to 2014 '. bSignificant associations $(P<0.05)$ in individual group comparison with the non-exposed group 'No ATD from 2005 to 2014 ': MMI in early pregnancy: none. PTU in early pregnancy: Ear (Q16-17); $P=0.008$, Urinary system (Q60-64); $P=0.03$. ATD before or after pregnancy: Others (Q85-99); $P=0.02$.

From this evaluation, it appeared that the pattern of birth defects after MMI and PTU exposure and in non-exposed children differed (Table 2).

\section{MMI exposure}

Among MMI-exposed children, none of the subgroups of malformations (Table 2) reached statistical significance in comparison with the non-exposed group. The most frequent type of malformations after MMI exposure was congenital heart defects $(n=6)$. Notably, these were all heart septal defects. The incidence of heart septal defects was significantly increased after MMI exposure
(Fig. 2, upper panel), and predominantly atrial septum

\begin{tabular}{|c|c|c|}
\hline & Children $(n)$ & Crude HR \\
\hline No ATD (reference) & 682343 & 1.0 \\
\hline MMI in early pregnancy & 162 & 0.83 \\
\hline PTU in early pregnancy & 218 & 0.79 \\
\hline ATD before or after pregnancy & 1551 & 1.10 \\
\hline
\end{tabular}
defects (ASD) (Fig. 2, lower panel). On the other hand, no significantly increased incidence of septal heart defects was observed in children exposed to PTU or in children born to mothers treated with ATD before or after pregnancy (Fig. 2, upper panel).

Malformations of genital organs were the second most frequent type of birth defects after MMI exposure $(n=5)$ and these were all observed in males. When analyses were restricted to boys, MMI exposure was associated with a significantly increased incidence of genital malformations $(P=0.04)$, whereas no increased incidence was observed in children exposed to PTU $(P=0.5)$ or born to mothers

Table 3 Crude and adjusted hazard ratio (HR) with 95\% confidence interval ( $95 \% \mathrm{Cl}$ ) for birth defects diagnosed before the age of two years in children born to mothers treated with methimazole (MMI) or propylthiouracil (PTU) in early pregnancy and in children born to mothers treated with antithyroid drugs (ATD) before or after, but not in pregnancy. The reference group is children born to mothers with no use of ATD in the study period.

\begin{tabular}{c}
\hline $95 \% \mathrm{Cl}$ \\
\hline (ref.) \\
$0.45-1.54$ \\
$0.46-1.35$ \\
$0.92-1.31$ \\
\hline
\end{tabular}

\begin{tabular}{c}
\hline Adjusted $\mathbf{H R}^{\mathbf{a}}$ \\
\hline 1.0 \\
0.72 \\
0.89 \\
1.11 \\
\hline
\end{tabular}

\begin{tabular}{c}
\hline $95 \% \mathrm{Cl}$ \\
\hline (ref.) \\
$0.37-1.41$ \\
$0.52-1.54$ \\
$0.92-1.33$ \\
\hline
\end{tabular}

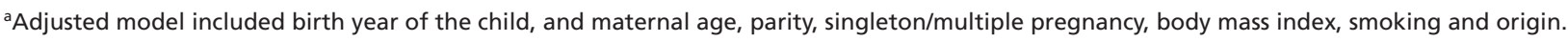


Cases/1000 live-born children

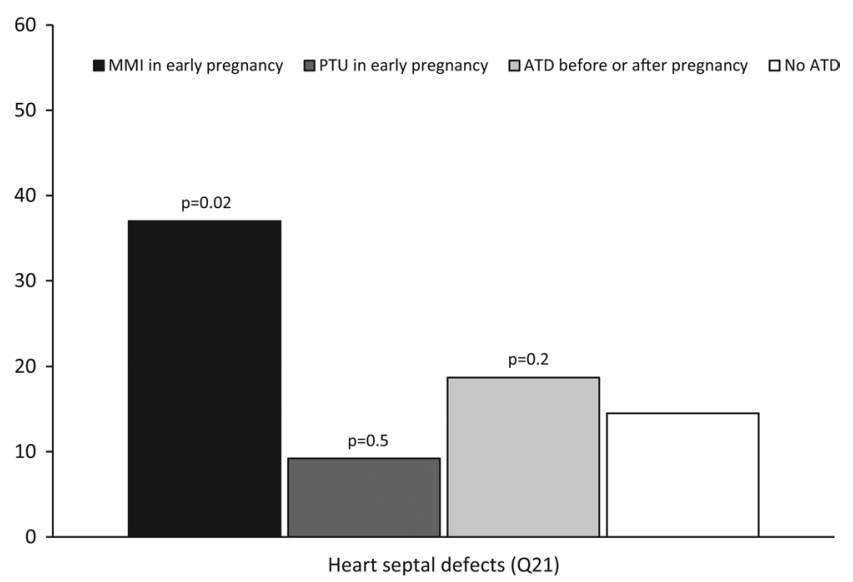

Cases/1000 live-born children

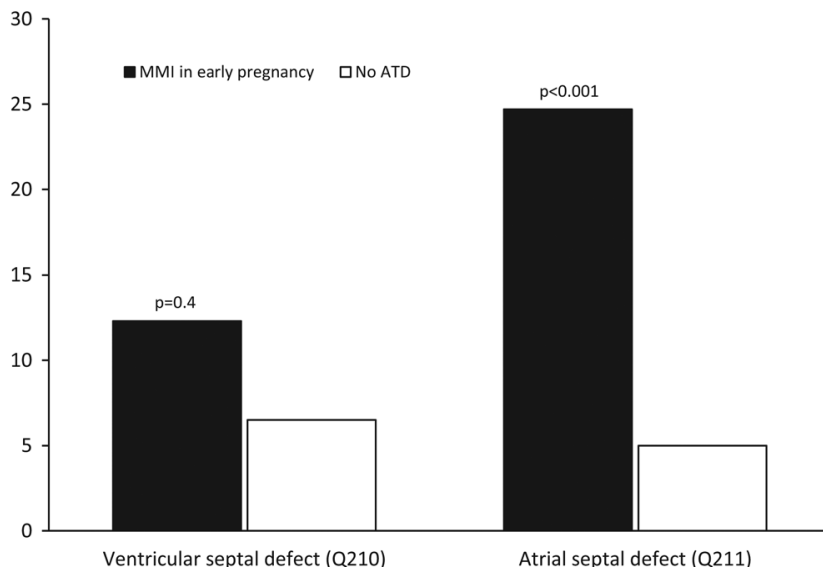

Figure 2

Cumulative incidence of septal heart defects diagnosed before the age of two years in children born to mothers treated with $\mathrm{MMI}$ in the early pregnancy ('MMI in early pregnancy') or PTU ('PTU in early pregnancy'), in children born to mothers treated with antithyroid drugs (ATD) before or after, but not in pregnancy ('ATD before or after pregnancy'), and in children born to mothers with no use of ATD in the study period ('No ATD'). $P$ values are result of chi-square test for comparison with 'No ATD'.

treated with ATD before or after pregnancy $(P=0.3)$ compared to non-exposed.

A notable observation among MMI-exposed children (Table 2) was the absence of cases of birth defects in the subgroups of severe malformations previously described in the MMI embryopathy. Thus, no cases of choanal atresia (Q30-38), esophageal atresia (Q39-45), omphalocele (Q79) or aplasia cutis (Q80-84) were observed after early pregnancy MMI exposure alone (Table 2).

\section{PTU exposure}

Among PTU-exposed children, the incidence of ear malformations $(P=0.008)$ and urinary system malformations $(P=0.03)$ were significantly increased compared with non-exposed (Table 2). The malformations observed after PTU exposure (Fig. 3) were confined to other malformations of the ear (Q17) including accessory auricle $(n=2)$ and obstructive urinary system malformations (Q62) including congenital hydronephrosis $(n=3)$. No cases of other ear malformations (Q17) was observed after MMI exposure or in children born to mothers treated with
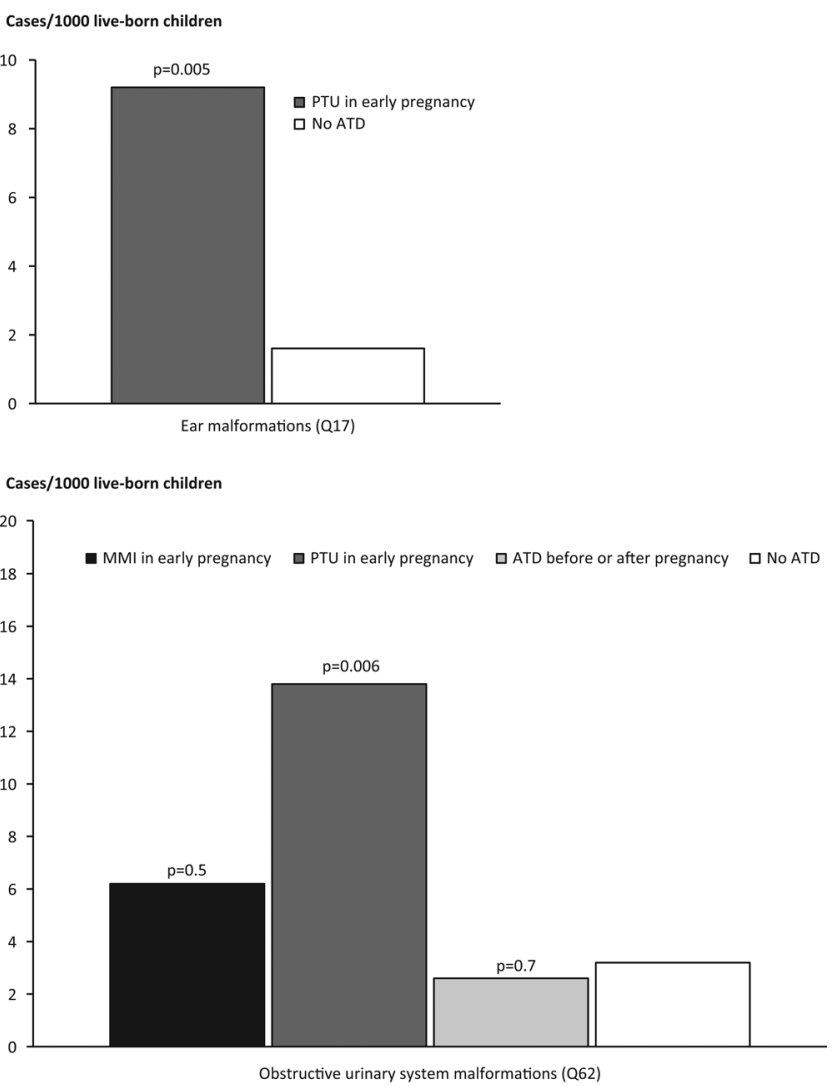

\section{Figure 3}

Cumulative incidence of ear malformations and obstructive urinary tract malformations diagnosed before the age of two years in children born to mothers treated with $\mathrm{MMI}$ in the early pregnancy ('MMI in early pregnancy') or PTU ('PTU in early pregnancy'), in children born to mothers treated with ATD before or after, but not in pregnancy ('ATD before or after pregnancy') and in children born to mothers with no use of ATD in the study period ('No ATD'). No cases of ear malformations (Q17) were observed in the 'MMI in early pregnancy' and the 'ATD before or after pregnancy' group. $P$ values are result of chi-square test for comparison with 'No ATD'. 
ATD before or after pregnancy (Fig. 3, upper panel). For malformations of the urinary system, the incidence was not significantly increased after early pregnancy MMI exposure or in children born to mothers treated with ATD before or after pregnancy (Fig. 3, lower panel).

Finally, two cases of malformations within the digestive system were observed in children exposed to PTU (Table 2), but comparison with non-exposed did not reach statistical significance $(P=0.06)$. The cases were malformations within the upper alimentary tract and the intestine. No malformations of the digestive system were observed after MMI exposure (Table 2), and the incidence in children born to mothers treated with ATD before or after pregnancy was similar to non-exposed $(0.3 \%, P=1.0)$.

\section{MMI and PTU exposure}

A subgroup of children $(n=66)$ were born to mothers who redeemed prescriptions of both MMI and PTU in the early pregnancy. In this group, four children (6.1\%) had birth defects diagnosed before the age of two years, which was not significantly different from the incidence among non-exposed $(P=0.6)$. The birth defects included choanal atresia $(n=1)$, ventricular septal defects (VSD) $(n=2)$, male genital malformations $(n=3)$, urinary system malformation $(n=1)$ and malformation of upper alimentary tract $(n=1)$. Although the number of exposed children was small, the findings indicated an increased incidence of choanal atresia $(P<0.001)$, VSD $(P=0.02)$ and male genital malformations $(P=0.03)$ compared with non-exposed. The majority of children $(n=62)$ were born to mothers who changed from MMI to PTU including three children with birth defects. The median time from pregnancy start to the shift from MMI to PTU was 41 days (interquartile range (IQR) 33-49 days) among the 59 children with no birth defects. Among children diagnosed with birth defects $(n=3)$, timing of shifting was significantly later (median 56 days (IQR 52-56 days), $P=0.03$ ) suggesting a longer period of MMI exposure.

\section{Sensitivity analysis}

The primary outcome was predefined as a diagnosis of birth defects in the child before the age of two years. In a post hoc sensitivity analysis, we obtained similar results when the follow-up period for a diagnosis of birth defects was restricted to one year (incidence of birth defects before one year of age in MMI exposed: 5.6\%, PTU exposed: $5.1 \%$, ATD before or after, but not in the pregnancy:
$6.7 \%$, non-exposed: $6.6 \%, P=0.8)$. The incidences of birth defects at one month of age were in MMI exposed: 1.2\%, PTU exposed: $2.8 \%$, ATD before or after, but not in the pregnancy: $2.9 \%$, non-exposed: $3.1 \%$, and not significantly different in overall comparison $(P=0.6)$ or for comparison of MMI-exposed vs non-exposed $(P=0.2)$ and PTU exposed vs non-exposed $(P=0.9)$.

\section{Discussion}

\section{Principal findings}

In a Swedish nationwide study, no overall increased incidence of birth defects was observed in children born to mothers treated with MMI or PTU in early pregnancy compared to non-exposed children and to children whose mother had been treated with ATD before or after, but not in pregnancy. However, for subtypes of birth defects, PTU and MMI were associated with types of birth defects previously described in a similar setting including ear and urinary system malformations after PTU exposure and heart septal defects after MMI exposure. On the other hand, severe malformations described in the MMI embryopathy were rarely observed in this study.

\section{Previous findings}

In 2002, a short report from Sweden raised concern about the use of MMI in early pregnancy (19). The authors had searched the Swedish MBR and the Swedish Register of Congenital Malformations in the period from 1995 to 2000 and reported four cases of children with severe malformations including esophageal atresia, omphalocele or choanal atresia after maternal treatment with 20-30 mg MMI/day in early pregnancy. In their letter (19), they cautioned against the use of MMI in women of reproductive age unless they used a safe birth control regime. Their report was in line with other case presentations about this time and the proposal of an MMI embryopathy (20). As reviewed by Mandel and Cooper in 2001 (21), no cases of malformations after PTU had been reported at this time, and PTU was preferred for the treatment of hyperthyroidism in pregnancy, but the MMI embryopathy was considered rare.

More recent studies have shown divergent results (22). Some studies showed no association $(8,10,12,13)$, whereas studies from Japan (9) and Denmark (11) have shown an increased risk of birth defects after the use of MMI in early pregnancy and the Danish study reported an 
increased prevalence of birth defects after PTU exposure (14). The present study was conceptualized to extend the burden of evidence, particularly the findings in the Danish study on birth defects after PTU exposure. The study was nationwide and register-based similar to the Danish study and similar definitions of exposure and outcome were used to ensure comparability and to reduce the risk of differential misclassification.

Overall, the present study showed no increased incidence of birth defects after maternal use of MMI or PTU in the early pregnancy. However, the study indicated associations with subtypes of malformations previously observed and described in the Danish study (11). MMI exposure was associated with a higher incidence of heart septal defects in both studies. The Danish study showed a dominant feature of VSD (18). ASD was the predominant subtype in this study, but VSD was observed among children exposed to both MMI and PTU. Previous reports have indicated that heart septal defect may be part of the MMI embryopathy (18). The clinical impact of such defect after ATD exposure needs to be addressed in further studies with longer follow-up; however, we noticed that the majority of children identified with heart septal defects after ATD exposure in the present study had registrations of multiple malformations. A notable difference between this study and the studies from Japan (9) and Denmark (11) was the absence of severe malformations indicative of the MMI embryopathy apart from one case of choanal atresia observed among children exposed to both MMI and PTU. A methodological limitation of the present study was the low frequency of MMI exposure. Furthermore, we studied all live-born children in Sweden, which may underestimate the frequency of severe malformations not leading to birth of a live-born child. Characteristic of the studies that found an association $(9,11)$ was the large number of MMI-exposed children ( $>1000$ children), whereas a similarity between the present study and other recent studies $(8,10,12,13)$ that found no association was the relatively low frequency of MMI exposure $(<200$ children) (22). In addition to sample size, many other aspects possibly determine differences between studies. Information on and definition of ATD exposure as well as outcomes of birth defects differ between studies. Furthermore, the hyperthyroidism of Graves' disease is linked to genetic and environmental factors (23) and the etiology of birth defects is similarly complex (24). Thus, other risk factors may also be of causal importance.

PTU exposure was associated with an increased incidence of ear malformations, and obstructive urinary tract defects in the present study, which is in line with the findings from the Danish study (14). The type of ear malformation differed slightly and the majority of Danish cases were diagnosed as preauricular sinus/fistula/cyst, whereas the Swedish cases included accessory auricle. Both types of defects arise from abnormal development of the auricle and are typically located anterior to the auricle $(25,26)$. The preauricular sinus/fistula/cyst typically presents as a small pit (25), whereas the accessory auricle typically presents as a skin-covered nodule (26). A shared feature was the registration of surgical procedures related to the disorder. The types of birth defects of the urinary system also showed similarities between the studies and were specified as obstructive defects of the urinary system.

The present study observed cases of gastrointestinal malformations after PTU exposure. A recent case report described congenital bands with intestinal malrotation in the child after maternal use of PTU in pregnancy (27). Another study described gastrointestinal malformations after exposure to untreated maternal hyperthyroidism (28). We had no information on maternal thyroid function or dose of ATD. Studies covering this information have shown divergent results $(9,29)$ and the role of these factors needs to be further addressed in future studies. In line with the Danish study (11), the present study showed no increased risk of birth defects in children born to mothers treated with ATD before or after, but not in pregnancy. Similarly, a previous study from Sweden (30) compared birth characteristics of children born to mothers previously treated for Graves' disease or toxic nodular goiter with children born to mothers with a history of surgical treatment for non-toxic goiter and showed no difference in the incidence of birth defects between these groups.

\section{Methodological comments}

The validity of the nationwide registers in Sweden is considered high $(15,16,17,31)$, and we defined exposure from redeemed prescriptions of drugs to reduce the risk of referral bias (32). We do not know if the women actually took the medicine, but compliance with drugs used for treatment of chronic disorders in pregnancy is considered high (33). We acknowledge that our study was limited by the low frequency of ATD exposure in early pregnancy, which is a challenge when the outcome under study is rare (22) and, thus, especially for the analyses of subtypes of birth defects. A lower incidence of hyperthyroidism in Sweden than that in Denmark is compatible with a historical lower iodine intake in Denmark $(34,35)$. Other potential 
aspects are differences in smoking habits (36) and in the clinical management of hyperthyroidism in women of reproductive age. Thus, the clinical practice in Sweden may favor definitive therapy before pregnancy in women of childbearing age. The incidence of birth defects observed in the present study was $8 \%$ in nonexposed children at the age of two years. We included diagnoses of both minor and major malformations from inpatient and outpatient visits in hospitals and in outpatient clinics. Thus, the majority were diagnosed at outpatient visits. The incidence when assessed at one month of age was $\sim 3 \%$ compatible when general figures $(24,37)$.

\section{Perspectives}

Recently revised clinical practice guidelines highlight the importance of detecting a pregnancy as early as possible and to perform any change in therapy before the critical window of exposure in early pregnancy (4, 5). Although the present study was challenged by a low frequency of ATD exposure, evaluation of children born to mothers who shifted from MMI to PTU in the early pregnancy indicated a potential risk associated with a relatively late shift in therapy, in line with a previous report (7).

Guidelines recommend the use of PTU for the treatment of maternal hyperthyroidism in early pregnancy, and also suggest the possibility of ATD withdrawal if the risk of relapse or worsening of hyperthyroidism is considered low $(4,5)$. The recommendation on ATD withdrawal is classified as a weak recommendation based on low-quality evidence and the risk of relapse after ATD withdrawal in pregnancy specifically has not been determined $(4,5)$. This study reports an increased incidence of subtypes of malformations after both MMI and PTU in line with previous reports, but future large studies are needed including evaluation of dose dependency and the potential impact of maternal hyperthyroidism and autoimmunity per se.

\section{Declaration of interest}

The authors declare that there is no conflict of interest that could be perceived as prejudicing the impartiality of the research reported.

\section{Funding}

This research did not receive any specific grant from any funding agency in the public, commercial or not-for-profit sector.

\section{Acknowledgements}

The authors acknowledge Prof. Peter Laurberg, Department of Endocrinology, Aalborg University Hospital who passed away during the preparation of the manuscript. Professor Peter Laurberg conceptualized and initiated the study and participated in the design and the initial discussion of the results. His contributions were invaluable and are greatly missed.

\section{References}

1 Andersen SL, Olsen J, Wu CS \& Laurberg P. Low birth weight in children born to mothers with hyperthyroidism and high birth weight in hypothyroidism, whereas preterm birth is common in both conditions: a Danish National Hospital Register study. European Thyroid Journal 20132 135-144. (doi:10.1159/000350513)

2 Andersen SL, Olsen J, Wu CS \& Laurberg P. Spontaneous abortion, stillbirth and hyperthyroidism: a Danish population-based study. European Thyroid Journal 20143 164-172. (doi:10.1159/000365101)

3 Andersen SL, Olsen J \& Laurberg P. Foetal programming by maternal thyroid disease. Clinical Endocrinology 201583 751-758. (doi:10.1111/ cen.12744)

4 Ross DS, Burch HB, Cooper DS, Greenlee MC, Laurberg P, Maia AL, Rivkees SA, Samuels M, Sosa JA, Stan MN et al. 2016 American Thyroid Association guidelines for diagnosis and management of hyperthyroidism and other causes of thyrotoxicosis. Thyroid 201626 1343-1421. (doi:10.1089/thy.2016.0229)

5 Alexander EK, Pearce EN, Brent GA, Brown RS, Chen H, Dosiou C, Grobman WA, Laurberg P, Lazarus JH, Mandel SJ et al. 2017 Guidelines of the American Thyroid Association for the diagnosis and management of thyroid disease during pregnancy and the postpartum. Thyroid 201727 315-389. (doi:10.1089/thy.2016.0457)

6 Andersen SL, Olsen J \& Laurberg P. Antithyroid drug side effects in the population and in pregnancy. Journal of Clinical Endocrinology and Metabolism 2016101 1606-1614. (doi:10.1210/jc.2015-4274)

7 Laurberg P \& Andersen SL. Antithyroid drug use in early pregnancy and birth defects. Time windows of relative safety and high risk? European Journal of Endocrinology 2014171 R13-R20. (doi:10.1530/eje14-0135)

8 Chen CH, Xirasagar S, Lin CC, Wang LH, Kou YR \& Lin HC. Risk of adverse perinatal outcomes with antithyroid treatment during pregnancy: a nationwide population-based study. BJOG 2011118 1365-1373. (doi:10.1111/j.1471-0528.2011.03019.x)

9 Yoshihara A, Noh J, Yamaguchi T, Ohye H, Sato S, Sekiya K, Kosuga Y, Suzuki M, Matsumoto M, Kunii Y, Watanabe N et al. Treatment of Graves' disease with antithyroid drugs in the first trimester of pregnancy and the prevalence of congenital malformation. Journal of Clinical Endocrinology and Metabolism 201297 2396-2403. (doi:10.1210/jc.2011-2860)

10 Korelitz JJ, McNally DL, Masters MN, Li SX, Xu Y \& Rivkees SA. Prevalence of thyrotoxicosis, antithyroid medication use, and complications among pregnant women in the United States. Thyroid 201323 758-765. (doi:10.1089/thy.2012.0488)

11 Andersen SL, Olsen J, Wu CS \& Laurberg P. Birth defects after early pregnancy use of antithyroid drugs: a Danish nationwide study. Journal of Clinical Endocrinology and Metabolism 201398 4373-4381. (doi:10.1210/jc.2013-2831)

12 Lo JC, Rivkees SA, Chandra M, Gonzalez JR, Korelitz JJ \& Kuzniewicz MW. Gestational thyrotoxicosis, antithyroid drug use and neonatal outcomes within an integrated healthcare delivery system. Thyroid 201525 698-705. (doi:10.1089/thy.2014.0434)

13 Gianetti E, Russo L, Orlandi F, Chiovato L, Giusti M, Benvenga S, Moleti M, Vermiglio F, Macchia PE, Vitale M et al. Pregnancy outcome in women treated with methimazole or propylthiouracil during pregnancy. Journal of Endocrinological Investigation 201538 977-985. (doi:10.1007/s40618-015-0281-z) 
14 Andersen SL, Olsen J, Wu CS \& Laurberg P. Severity of birth defects after propylthiouracil exposure in early pregnancy. Thyroid $2014 \mathbf{1 0}$ 1533-1540. (doi:10.1089/thy.2014.0150)

15 The Swedish Centre for Epidemiology, The National Board of Health and Welfare. The Swedish Medical Birth Register - a summary of content and quality 2003 2003-112-3 1-33.

16 Wettermark B, Hammar N, Fored CM, Leimanis A, Otterblad Olausson P, Bergman U, Persson I, Sundstrom A, Westerholm B \& Rosen M. The new Swedish Prescribed drug register - opportunities for pharmacoepidemiological research and experience from the first six months. Pharmacoepidemiology and Drug Safety 200716 726-735. (doi:10.1002/pds.1294)

17 Ludvigsson JF, Andersson E, Ekbom A, Feychting M, Kim JL, Reuterwall C, Heurgren M \& Olausson PO. External review and validation of the Swedish national inpatient register. BMC Public Health 201111450. (doi:10.1186/1471-2458-11-450)

18 Andersen SL \& Laurberg P. Antithyroid drugs and congenital heart defects: ventricular septal defect is part of the methimazole/ carbimazole embryopathy. European Journal of Endocrinology 2014171 C1-C3. (doi:10.1530/EJE-14-0524)

19 Karlsson FA, Axelsson O \& Melhus H. Severe embryopathy and exposure to methimazole in early pregnancy. Journal of Clinical Endocrinology and Metabolism 200287 947-949. (doi:10.1210/jcem.87.2.8126)

20 Clementi M, Di Gianantonio E, Pelo E, Mammi I, Basile RT \& Tenconi R. Methimazole embryopathy: delineation of the phenotype. American Journal of Medical Genetics 199983 43-46. (doi:10.1002/ (SICI)1096-8628(19990305)83:1<43::AID-AJMG8>3.0.CO;2-C)

21 Mandel SJ \& Cooper DS. The use of antithyroid drugs in pregnancy and lactation. Journal of Clinical Endocrinology and Metabolism 200186 2354-2359. (doi:10.1210/jcem.86.6.7573)

22 Laurberg P \& Andersen SL. Antithyroid drug use in pregnancy and birth defects: why some studies find clear associations, and some studies report none. Thyroid 201525 1185-1190. (doi:10.1089/thy.2015.0182)

23 Smith TJ \& Hegedus L. Graves' disease. New England Journal of Medicine 2016375 1552-1565. (doi:10.1056/NEJMra1510030)

24 Moore KL, Persaud TVN \& Torchia MG. Human birth defects. In The Developing Human: Clinically Oriented Embryology, edn 9, ch 20, pp 471-501. Eds KL Moore, TVN Persaud \& MG Torchia. Philadelphia: Saunders/Elsevier, 2013.

25 Tan T, Constantinides H \& Mitchell TE. The preauricular sinus: a review of its aetiology, clinical presentation and management. International Journal of Pediatric Otorhinolaryngology 200569 1469-1474. (doi:10.1016/j.ijporl.2005.07.008)

26 Wong PY, Laing T \& Milroy C. One-stop outpatient management of accessory auricle in children with titanium clip. Plastic Surgery International 20142014 780394. (doi:10.1155/2014/780394)
27 Leung AA, Yamamoto J, Luca P, Beaudry P \& McKeen J. Congenital bands with intestinal malrotation after propylthiouracil exposure in early pregnancy. Case Reports in Endocrinology 20152015789762. (doi:10.1155/2015/789762)

28 Seoud M, Nassar A, Usta I, Mansour M, Salti I \& Younes K. Gastrointestinal malformations in two infants born to women with hyperthyroidism untreated in the first trimester. American Journal of Perinatology 200320 59-62. (doi:10.1055/s-2003-38320)

29 Momotani N, Ito K, Hamada N, Ban Y, Nishikawa Y \& Mimura T. Maternal hyperthyroidism and congenital malformation in the offspring. Clinical Endocrinology 198420 695-700. (doi:10.1111/j.1365-2265.1984.tb00119.x)

30 Ohrling H, Torring O, Yin L, Iliadou AN, Tullgren O, AbrahamNordling M, Wallin G, Hall P \& Lonn S. Decreased birth weight, length, and head circumference in children born by women years after treatment for hyperthyroidism. Journal of Clinical Endocrinology and Metabolism 201499 3217-3223. (doi:10.1210/ jc.2014-1168)

31 Wallerstedt SM, Wettermark B \& Hoffmann M. The first decade with the Swedish prescribed drug register - a systematic review of the output in the scientific literature. Basic and Clinical Pharmacology and Toxicology 2016119 464-469. (doi:10.1111/bcpt.12613)

32 Carle A, Pedersen IB, Perrild H, Ovesen L, Jorgensen T \& Laurberg P. High age predicts low referral of hyperthyroid patients to specialized hospital departments: evidence for referral bias. Thyroid 201323 1518-1524. (doi:10.1089/thy.2013.0074)

33 Olesen C, Sondergaard C, Thrane N, Nielsen GL, de Jong-vanden Berg L, Olsen J \& EuroMAP Group. Do pregnant women report use of dispensed medications? Epidemiology 200112 497-501. (doi:10.1097/00001648-200109000-00006)

34 Abraham-Nordling M, Bystrom K, Torring O, Lantz M, Berg G, Calissendorff J, Nystrom HF, Jansson S, Jorneskog G, Karlsson FA et al. Incidence of hyperthyroidism in Sweden. European Journal of Endocrinology 2011165 899-905. (doi:10.1530/ EJE-11-0548)

35 Carle A, Pedersen IB, Knudsen N, Perrild H, Ovesen L, Rasmussen LB \& Laurberg P. Epidemiology of subtypes of hyperthyroidism in Denmark: a population-based study. European Journal of Endocrinology 2011164 801-809. (doi:10.1530/EJE-10-1155)

36 Andersen SL, Olsen J, Wu CS \& Laurberg P. Smoking reduces the risk of hypothyroidism and increases the risk of hyperthyroidism: evidence from 450842 mothers giving birth in Denmark. Clinical Endocrinology 201480 307-314. (doi:10.1111/cen.12279)

37 The Swedish Centre for Epidemiology, The National Board of Health and Welfare Registration of Congenital Malformations in the Swedish Health Registers 2004 2004-112-1 1-10.

Received 20 April 2017

Revised version received 2 June 2017

Accepted 31 July 2017 